\title{
A COPPER 3.9 GHZ TM110 CAVITY FOR EMITTANCE EXCHANGE*
}

\author{
T.W. Koeth", Rutgers University, Piscataway, NJ 08854, U.S.A. \\ R.P. Fliller III, L. Bellantoni, D.A. Edwards, H.T. Edwards, FNAL, Batavia, IL 60510 U.S.A
}

\section{Abstract}

An experiment is being developed at the FNAL Photoinjector Lab to demonstrate the exchange of longitudinal emittance with a transverse horizontal emittance. The longitudinal electric field of a $\mathrm{TM}_{110}$ cavity vanishes on axis and increases linearly with transverse displacement. This "shearing" electric field is pivotal to the exchange. The design of this $\mathrm{TM}_{110}$ cavity is a variant of the Fermilab $3.9 \mathrm{GHz}$ superconducting deflecting mode cavity; however, the cavity was constructed of OFHC copper. The authors report on the construction, field flatness, polarization and high power testing of a $\mathrm{TM}_{110}$ cavity.

\section{INTRODUCTION}

An experiment is being developed at Fermilab to demonstrate transverse-longitudinal phase space interchange in the technique proposed by Kim [1]. Further discussion of the overall experiment and its physics basis may be found in the accompanying paper by Fliller et al [2] and in the references cited therein. The $\mathrm{TM}_{110} \mathrm{RF}$ cavity required by the process is based on the Fermilab 3.9 GHz superconducting deflecting mode cavity originally designed for an RF separated beam [3]. However, the present cavity was constructed with OFHC copper to avoid the complexities of a liquid helium cryogenic system.

\section{CAVITY REQUIREMENTS}

The cavity transfer matrix in a 5-cell thick lens pillbox reads[4]:

$$
M_{c}=\left[\begin{array}{cccc}
1 & 5 L & 5 k L / 2 & 0 \\
0 & 1 & k & 0 \\
0 & 0 & 1 & 0 \\
k & 5 k L / 2 & 17 k^{2} L / 20 & 1
\end{array}\right]
$$

where $k$ is the cavity strength defined as

$$
k=\frac{e V_{L}}{a E},
$$

and $E$ is the beam energy (16MeV in our case), $V_{L}$ is the longitudinal kick needed a distance $a$ offset from the axis. This differs from a single cell pillbox by an increase in the $\mathrm{M}_{43}$ element, which yields to a larger degradation in the exchange. For optimum emittance exchange,

$$
k=\frac{-1}{\eta}
$$

where $\eta=0.33 \mathrm{~m}$ is the dispersion at the cavity.

Inserting the other parameters of our experiment, the integrated longitudinal kick at $1 \mathrm{~mm}$ offset from the axis is approximately $45 \mathrm{keV}$. The power requirement is determined from:

$$
V_{L}(r)=\sqrt{2\left(\frac{R}{Q}\right)^{\prime} P Q_{o} \frac{4 \beta}{(1+\beta)^{2}}} \frac{r \omega}{c} .
$$

We have access to an $80 \mathrm{~kW} 3.9 \mathrm{GHz}$ klystron, thus we have designed our cavity to use this klystron in phase lock with the FNAL 1.3 GHz Photoinjector. Losses in the transmission line further reduce the available power and mode spacing limits this structure to five cells. To compensate for these limitations it was found necessary to increase the copper cavity's Q above that at 293K.

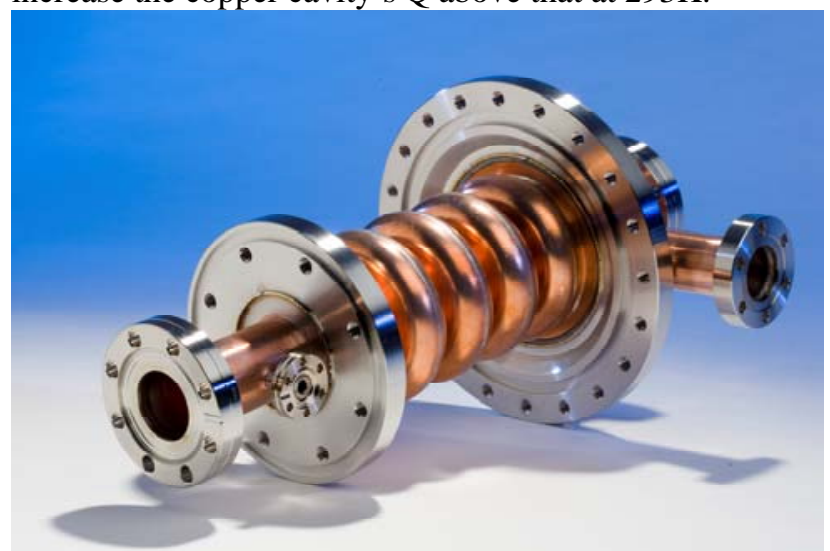

Fig.1 Copper 5-cell 3.9GHz $\mathrm{TM}_{110}$ cavity.

\section{CAVITY CONSTRUCTION}

Since the field requirement is modest it was decided to construct the cavity out of OFHC copper. The higher required $\mathrm{Q}$ is achieved by cooling the cavity with liquid nitrogen; thus a simple cryogenic jacket was incorporated into the design. With the exception of one end-half cell, the half cells were all formed by stamping $2.2 \mathrm{~mm}$ thick OFHC copper disks by Advanced Energy Systems with the same dies used for Niobium production. The half cells were brazed at the iris, forming dumbbells. The dumbbells were electrically characterized and trimmed at the equator for the $\pi$ mode to be $3.885 \mathrm{GHz}$ in anticipation that the frequency would raise $12 \mathrm{MHz}$ due to thermal contraction at $\mathrm{IN}_{2}$ temperature, and providing 3 $\mathrm{MHz}$ for field flatness tuning.

After trimming to the proper frequencies, each cell was found to be about 4\% longer than free space halfwavelength at $3.9 \mathrm{GHz}$, which corresponds to a $7^{\circ}$ phase slip per cell. This does not pose a serious problem for our 5-cell cavity; proper low level RF phase control and increased RF power can compensate. The longer suboptimum cell length is a result of the different thermal contraction between copper and $\mathrm{Nb}$ and the necessity to etch the Nb cavity. Both effects lead to needing to tune this cavity to lower frequency and longer length to obtain the correct operating frequency. 
A final braze of the dumbbells and end assemblies completed the structure. Because of the close proximity of the centerline of the coaxial input coupler $(20 \mathrm{~mm})$ to the iris of the end cell, the liquid nitrogen jacket, input coupler, and beam pipe required that they be machined out of a single piece of copper. An RF pick-up probe was mounted on the beam pipe on the non-input coupler end. In order to protect their sealing surfaces all of the stainless steel flanges were welded to previously brazed copper-to-stainless steel transitions only after the final braze.

The thin wall fully annealed copper structure needed careful handling and constraints when pulling vacuum to prevent deformation and detuning.

The $\mathrm{IN}_{2}$ jacket is a simple demountable stainless steel vessel which incorporates a bellows bridged by 3 pairs of lugs stiffened by threaded rods. The threaded rods provide both a method of fine tuning the cavity and the rigidity to constrain the cavity against vacuum forces. The $\mathrm{IN}_{2}$ jacket is sealed to the $\mathrm{IN}_{2}$ flanges (visible in Fig.1) with all metal aluminium "diamond" seals.

Thermal insulation is easily provided by three layers of polyethylene foam cut to intimately contour the $\mathrm{lN}_{2}$ jacket, stiffening lugs, and plumbing fixtures. A polyethylene sheet is wrapped around the assembly, hermetically sealed with tape, and purged with dry nitrogen to prevent ice build-up.

\section{Field Flatness Tuning}

Cavity field flatness was achieved by the iterative process of presenting an identical perturbation to each of the five cells while monitoring the structure's frequency response and tuning individual cells. This was performed with a typical bead pull setup using a metal bead run down the electrical axis of the cavity. The initial peak RMS field flatness was about $20 \%$, final flatness was less than 2.6\%, as Fig.2 shows, which is within the systems resolution.

After several thermal cycles, the cavity field flatness was measured again. A slight deformation occurred which caused the flatness to rise to $4.5 \%$, still considered acceptable.

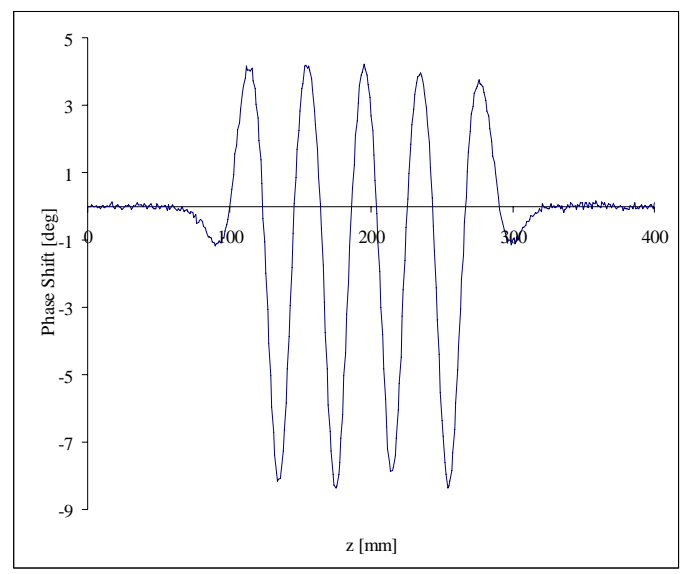

Fig.2 Final field flatness profile, flatness was $2.6 \%$

\section{$\mathbf{L N}_{2}$ COOL DOWN}

Cavity cool down to date has been extremely simple. A 160 liter pressure building dewar is connected to the liquid nitrogen jacket by means of an vacuum insulated line. A pressure relief valve, set to 18psia, is attached with a tee in line prior to entrance of the $\mathrm{lN}_{2}$ jacket to prevent an overpressure situation from deforming the cavity. A second port on the $\mathrm{IN}_{2}$ jacket exhausts the liquid into a heat exchanger, and the resulting nitrogen gas is vented to the atmosphere. A flow meter is placed in the gaseous portion of the vent line to quantify the nitrogen flow. The cavity temperature is monitored by means of a thermocouple fixed to an exposed portion of the cavity copper near the input coupler.

The dewar pressure and liquid exhaust valve are iteratively adjusted while monitoring the flow meter and temperature. The $\mathrm{lN}_{2}$ flow is set just below threshold of the relief valve for initial cool down. Cool down from room temperature to $80 \mathrm{~K}$ takes about 1 hour. When the cavity temperature indicates that the jacket is full of liquid, the flow is throttled back to read 350 Standard Cubic Feet per Hour on the flow meter. Once at steady state, the system typically runs for 6 hours without need for adjustment.

Slight pressure fluctuation in the system does cause the cavity's resonant frequency to shift $\pm 40 \mathrm{kHz}$ with a time constant measured in seconds; pressure regulation improvements are being developed to halve this shift.

\section{RF MEASUREMENTS}

\section{Polarization}

Unlike typical accelerating cavities, that have azimuthally symmetric fields, the dipole field exploited in the $\mathrm{TM}_{110}$ structure necessarily has two polarizations which are azimuthally dependant. Their orientations need to be fixed and frequencies separated. Typically, to orient the polarization a dimple is placed in a row on each cell, however, it was found that the input coupler perturbation of the structure was sufficient to force the orientation of the mode. The $\pi$ mode polarization separation is greater than $5 \mathrm{MHz}$. Generally the lower frequency polarization is used; however, the dominant polarization forced by the input coupler was the higher frequency. Again, the operating frequency could have been lowered past that of the undesired polarization by dimpling; but at the risk of cracking a braze joint.

The cell to cell polarization was also measured through a bead pull technique. First the electrical center of the cavity was determined by a series of dielectric bead pulls in the plane of the peak $E_{z}$ field. Then the dielectric bead was pulled through the structure at eight locations $10 \mathrm{~mm}$ offset from the electrical center in a $45^{\circ}$ incremental circular pattern. The bead perturbations induced phase shifts that were measured and fit to the function $E^{2} \cos ^{2}(\theta)$. Fig.3 shows the resulting fit of the 8 points to cells 2,3 , and 4 . Since the off axis electric energy density does not go through a minimum in the 
equatorial plane of the end cells, the polarization could not be determined by this bead pull technique; their orientations were directly measured with small loop probes inserted on axis through their respective beam pipes. The end cell polarizations were found to be aligned with the body cells.

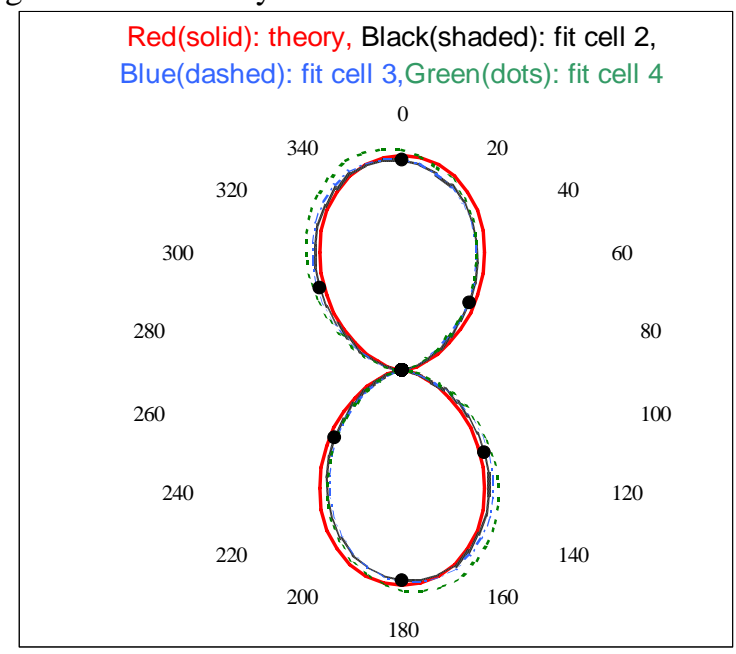

Fig.3 Cells 2, 3, and 4 polarizations compared with ideal.

\section{Low Power $\mathrm{IN}_{2}$ Cold Tests}

During low power testing the 5-cell was outfitted with an adjustable model of the input coupler. The input coupler had sufficient adjustment to go through unity coupling at both warm and cold temperatures to measure the increase in $\mathrm{Q}_{0}$. Unity coupling was empirically determined by noting the penetration that caused the minimum insertion loss in an $\mathrm{S}_{21}$ measurement. Fig.4 shows the data taken both warm and cold. The cavity was thermally cycled to verify reproducibility. As expected, thermal contraction increased the frequency approximately $11.8 \mathrm{MHz}$ to $3.89930 \mathrm{GHz}$.

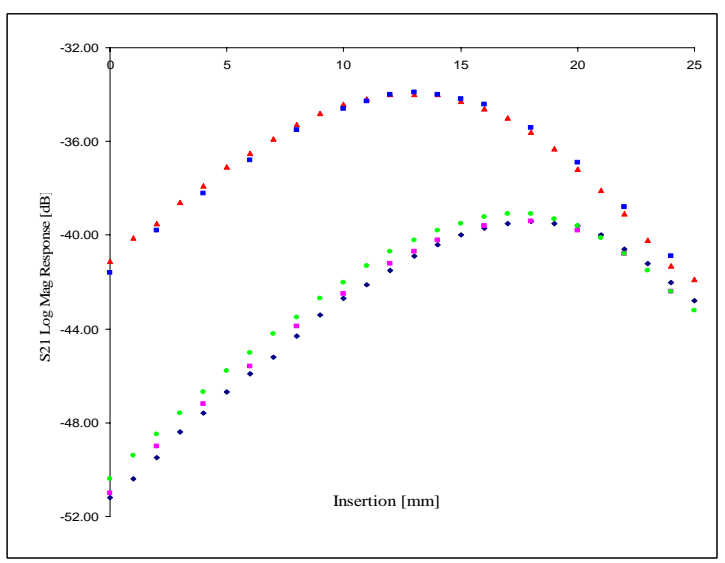

Fig. $4 \beta=1$ positions for cavity cold (upper) and warm.

The first thermal cycle slightly deformed the cavity to permanently increase the frequency $500 \mathrm{kHz}$, certainly within tuneable range; subsequent thermal cycles have reproducibly brought the resonant frequency to within 40 $\mathrm{kHz}$. The initial deformation is blamed for the reduction of field flatness (seen in radial amplitudes in Fig.3); the cell-to-cell polarization did not vary.
$\mathrm{Q}_{0}=14,800$ at $293 \mathrm{~K}$ and increased to 35,600 at $\mathrm{lN}_{2}$ temperature. This increase is in agreement with the change in $\mathrm{Cu}$ conductivity between room temperature and $\mathrm{IN}_{2}$. The location for $\beta=1$ coupling cold corresponded to $\beta=0.4$ warm.

\section{High Power Warm Conditioning}

The high power input coupler utilized is one from another $3.9 \mathrm{GHz}$ project. Its length, and thus the coupling coefficient, is fixed. An extension of appropriate length was installed on the center conductor of the fixed length high power input coupler to match the empirically found unity coupling, $\beta=1$, length.

The test input coupler was replaced with the lengthcorrected high power input coupler. This input coupler was brand new and was not previously conditioned. Cavity/coupler on resonance conditioning started with the $100 \mu$ sec pulse at low power level. Electron pickup probes mounted on the input coupler were interlocked to the klystron modulator. Approximately a dozen breakdown events occurred during the conditioning, which typically occurred in conjunction with an incremental RF power level increase. The conditioning concluded after several hours of dwelling the maximum power level available $(45 \mathrm{~kW}$ at $3.889 \mathrm{GHz})$ without event. Analysis of the waveforms indicated $\beta=0.4$, as was expected.

\section{High Power $I_{2}$ Cold Tests}

After warm conditioning, the cavity was cooled to $\mathrm{lN}_{2}$ temperature and a rectangular RF pulse was applied to the cavity. The power was brought up slowly without any noticeable break down events. Analysis of the RF waveforms indicated that the system was still undercoupled at $\beta=0.6$. The measured cavity decay curve yielded $\mathrm{Q}_{\text {loaded }}$ of 22,000 confirming a lower $\beta$ than expected. This discrepancy may have arisen from an over pressure situation which modified the field flatness and thus coupling; it is being investigated. With the available power $(54 \mathrm{~kW}$ at $3.900 \mathrm{GHz})$ and with $\beta=0.6$ we infer an integrated $\mathrm{V}_{\mathrm{L}}$ of $57 \mathrm{kV} 1 \mathrm{~mm}$ offset from the axis.

\section{FUTURE STEPS}

A DESY 1.3 GHz low level RF system has been modified with up and down converters to control the 3.9 $\mathrm{GHz}$ system. It is presently being debugged. The cavity will be installed in the Photoinjector beam in July 2007.

\section{REFERENCES}

[1] K.-J. Kim and A. Sessler, AIP Conf. Proc. 821, 115 (2006).

[2] R. P. Fliller, et. al "Transverse to Longitudinal Emittance Exchange beamline at the A0 Photoinjector”, PAC'07, Albuquerque, June 2007

[3] M. McAshan and R. Wanzenberg, FNAL TM-2144, 22 March 2001.

[4] D.A. Edwards. Unpublished 
